\title{
Caveat of measuring perfusion indexes using intravoxel incoherent motion magnetic resonance imaging in the human brain
}

\author{
Wen-Chau Wu $\cdot$ Ya-Fang Chen $\cdot$ Han-Min Tseng • \\ Shun-Chung Yang $\cdot$ Pei-Chi My
}

Received: 1 November 2014 /Revised: 22 January 2015 / Accepted: 3 February 2015 /Published online: 19 February 2015

(C) The Author(s) 2015. This article is published with open access at Springerlink.com

\begin{abstract}
Objectives To numerically and experimentally investigate the robustness of intravoxel incoherent motion (IVIM) magnetic resonance imaging in measuring perfusion indexes in the human brain.

Methods Eighteen healthy volunteers were imaged on a $3 \mathrm{~T}$ clinical system. Data of IVIM imaging (12 b-values ranging from 0 to $1000 \mathrm{~s} / \mathrm{mm}^{2}, 12$ repetitions) were fitted with a biexponential model to extract blood volume fraction (f) and pseudo-diffusion coefficient $\left(\mathrm{D}^{*}\right)$. The robustness of measurement was assessed by bootstrapping. Dynamic susceptibility contrast (DSC) imaging and arterial spin-labelling (ASL) imaging were performed for cross-modal comparison. Numerical simulations were performed to assess the accuracy and precision of $\mathrm{f}$ and $\mathrm{D}^{*}$ estimates at varied signal-to-noise ratio $\left(\mathrm{SNR}_{\mathrm{b} 1000}\right)$.

Results Based on our experimental setting $\left(\mathrm{SNR}_{\mathrm{b} 1000} \sim 30\right)$, the average error/variability is $\sim 5 \% / 25 \%$ for $\mathrm{f}$ and $\sim 100 \% /$
\end{abstract}

W.-C. $\mathrm{Wu}(\triangle)$

Graduate Institute of Oncology, National Taiwan University,

Taipei, Taiwan

e-mail:wenchau@ntu.edu.tw

W.-C. Wu

Graduate Institute of Clinical Medicine, National Taiwan University, Taipei, Taiwan

W.-C. $\mathrm{Wu}$

Graduate Institute of Biomedical Electronics and Bioinformatics, National Taiwan University, Taipei, Taiwan

W.-C. Wu $\cdot$ Y.-F. Chen $\cdot$ S.-C. Yang $\cdot$ P.-C. My

Department of Medical Imaging, National Taiwan University

Hospital, Taipei, Taiwan

H.-M. Tseng

Department of Neurology, National Taiwan University Hospital,

Taipei, Taiwan
$30 \%$ for $D^{*}$ in gray matter, and $\sim 10 \% / 50 \%$ for f and $\sim 300 \% /$ $60 \%$ for $\mathrm{D}^{*}$ in white matter. Correlation was found between $\mathrm{f}$ and DSC-derived cerebral blood volume in gray matter $(\mathrm{r}=$ $0.29-0.48$ across subjects, $\left.p<10^{-5}\right)$, but not in white matter. No correlation was found between f-D* product and ASLderived cerebral blood flow.

Conclusions $\mathrm{f}$ may provide noninvasive measurement of cerebral blood volume, particularly in gray matter. $\mathrm{D}^{*}$ has limited robustness and should be interpreted with caution.

Key Points

- A minimum $S N R_{b 1000}$ of 30 is recommended for reliable IVIM imaging.

- $f$ may provide noninvasive measurement of cerebral blood volume.

- $f$ correlates with $C B V_{D S C}$ in gray matter.

- There is no correlation between $f D^{*}$ and $C B F_{A S L}$.

- $D^{*}$ has limited robustness and should be interpreted with caution.

Keywords Magnetic resonance imaging · Intravoxel incoherent motion $\cdot$ Perfusion $\cdot$ Brain $\cdot$ Imaging

\section{Introduction}

Microvascular blood flow, also known as perfusion, plays an important role in regulating physiology. In the brain, perfusion indexes such as cerebral blood flow $(\mathrm{CBF})$ and cerebral blood volume (CBV) have been shown useful in the diagnosis and prognosis of neurological diseases [1-3].

To date, three major magnetic resonance (MR) imaging techniques are available for cerebral perfusion measurement: dynamic susceptibility contrast (DSC) imaging [4], arterial spin-labelling (ASL) imaging [5], and 
intravoxel incoherent motion (IVIM) imaging [6]. With DSC imaging, images are repeatedly acquired to trace the signal evolution following the passage of intravenously administered paramagnetic contrast agent. Multiple perfusion indexes (e.g., CBV, CBF, mean transit time, and time to maximum) can be derived from the temporal dynamics [7-9]. Except for being impracticable in subjects with impaired renal function [10, 11], DSC imaging imposes little invasiveness and has been routinely performed in many hospitals. With ASL imaging, flow contrast is generated by using radiofrequency pulses tailored to label the protons electromagnetically in arterial blood. Most existing ASL methods provide CBF measurement only [12], although variants have been proposed to estimate arterial CBV [13] and arterial transit time [14]. The caveat of ASL imaging is the inherently low signal-to-noise ratio (SNR). First proposed by Le Bihan et al [6], IVIM imaging measures the signal drop caused by intravoxel incoherent motion at varied magnitudes of diffusion weighting (quantified by b-values). Perfusion indexes are derived by modelling the signal attenuation as a composite outcome of interstitial water diffusion (characterized by diffusion coefficient D) and intravascular capillary blood flow:

$$
\frac{S(b)}{S_{0}}=(1-f) \exp (-b D)+f \exp \left(-b D^{*}\right)
$$

where $S_{0}$ is the signal intensity obtained with $b=0$. D* is the pseudo-diffusion coefficient to account for the capillary blood flow that takes up a volume fraction $\mathrm{f}$. IVIM imaging has been applied in several body organs [15-18], and was assessed for measurement sensitivity in the rat brain [19] and recently in the healthy human brain [20]. Nonetheless, feasibility study remains relatively scarce in the human brain $[6,21]$.

As compared with its counterpart techniques, IVIM imaging does not rely on tracer delivery (e.g., transit of arterial protons for ASL imaging and gadolinium chelates for DSC imaging). This is a desirable feature as bolus dispersion and elongated transit time that stem from variable or abnormal flow dynamics or routes can confound tracer-based perfusion imaging. For example, ASL measurement of white matter perfusion has been challenging mainly because of the long transit time [22]. On the other hand, previous studies suggest that IVIM imaging is SNR demanding $[23,24]$ although the relationship between SNR and perfusion that can be reliably measured remains unclear. The purpose of this study was to numerically and experimentally investigate the robustness of IVIM MR imaging in measuring perfusion indexes in the human brain.

\section{Materials and methods}

\section{Subjects}

The institutional review board approved this study. Eighteen healthy volunteers (eight women, 10 men; age $=21-36$ years) were recruited. Written informed consent was obtained from all subjects beforehand.

\section{MR imaging}

All MR imaging was performed on a 3-Tesla whole body clinical system (Tim Trio, Siemens, Erlangen, Germany). The body coil was used to transmit radiofrequency pulses. A 12-channel phased-array head coil was used to receive signals. IVIM/ASL/DSC images were obtained from the same location (field of view $=200 \mathrm{~mm}$, in-plane matrix $=128 \times 128$, GRAPPA acceleration factor $=2$, slice thickness $=5 \mathrm{~mm}, 12$ axial slices, inter-slice gap $=1 \mathrm{~mm}$ ). Specifically, IVIM imaging was based on a single-shot, twice-refocused, spin-echo echo-planar readout $(\mathrm{TR}=2 \mathrm{~s}, \mathrm{TE}=100 \mathrm{~ms}, 12$ repetitions after a dummy scan, diffusion time $=42 \mathrm{~ms}$, ratio of diffusion time to gradient duration $\sim 1$ ). Diffusion weighting was applied along three orthogonal directions (in separate scans) with 12 b-values: $0,15,30,45,60,100,250,400,550,700,850,1$, $000 \mathrm{~s} / \mathrm{mm}^{2}$. The maximum b-value was chosen such that SNR was adequate and non-Gaussian diffusion was not prominent. ASL imaging was based on the pseudocontinuous labelling scheme [25] $(\mathrm{TR}=4.4 \mathrm{~s}, \mathrm{TE}=18 \mathrm{~ms}$, labelling duration $=2 \mathrm{~s}$, post-labelling delay $=1.5 \mathrm{~s}, 50$ pairs of tag and control images, single-shot gradient-echo echo-planar readout). Reference scans were included for coil sensitivity correction and labelling efficiency calibration [26]. For DSC imaging, single-shot gradient-echo echo-planar images were repeatedly acquired $(\mathrm{TR}=1 \mathrm{~s}, \mathrm{TE}=25 \mathrm{~ms}, 120$ measurements). At the 20th acquisition, a bolus of $0.1 \mathrm{mmol} / \mathrm{kg}$ bodyweight Gadobutrol (Gadovist, $1.0 \mathrm{mmol} / \mathrm{ml}$ ) was intravenously administered followed by a $15-\mathrm{ml}$ saline flush, at a rate of $4 \mathrm{ml} / \mathrm{s}$ using a power injector. T1-weighted anatomic images were acquired by using three-dimensional magnetization prepared rapid gradient echo. ASL imaging and IVIM imaging were performed back-to-back and in a semi-random order across subjects but always before DSC imaging.

\section{Data processing}

To extract IVIM indexes, a two-step fitting procedure was performed voxel by voxel based on equation (1). D was first obtained by fitted the signals obtained with $b=400-1,000 \mathrm{~s} /$ $\mathrm{mm}^{2}$ to a mono-exponential function given that perfusion effect is negligible in this regime [24]. The signals obtained with the rest of $b$-values were then fitted to equation (1) to extract $f$ 
and $\mathrm{D}^{*}$. Data obtained with different directions of diffusion encoding were processed separately and then averaged.

Following the procedure summarized in [26], the ASL images were corrected for spatial variation of coil sensitivity, pair-wise subtracted, averaged, and then converted to $\mathrm{CBF}_{\mathrm{ASL}}$ maps. Following the procedure summarized in [27], DSC signal time curves were converted to concentration time curves and fitted with a gamma-variate function to remove recirculation. $\mathrm{CBV}_{\mathrm{DSC}}$ was then calculated by dividing the timeintegral of concentration in tissue by the time-integral of arterial input function.

All echo-planar images were averaged to create a local template for each subject. The T1-weighted anatomic images were voxel-wise calculated for the probability of belonging to
Fig. 1 Simulated variance of IVIM-derived diffusion coefficient (D), blood volume fraction (f), and pseudo-diffusion coefficient $\left(\mathrm{D}^{*}\right)$. Gray matter and white matter are shown in subfigures (a) and (b), respectively. For a given signal-to-noise ratio at $\mathrm{b}=1,000 \mathrm{~s}$ $\mathrm{mm}^{2}\left(\mathrm{SNR}_{\mathrm{b} 1000}\right)$, variance was computed in terms of the coefficient of variation $(\mathrm{CV})$ from 1,000 random samples, for each combination of $\mathrm{f}(0.01$ to 0.20 , in steps of 0.01$)$ and $D *$ to $D$ ratio (1 to 50 , in steps of 1 ). Two levels of $\mathrm{SNR}_{\mathrm{b} 1000}$ are shown (30 and 100)

a

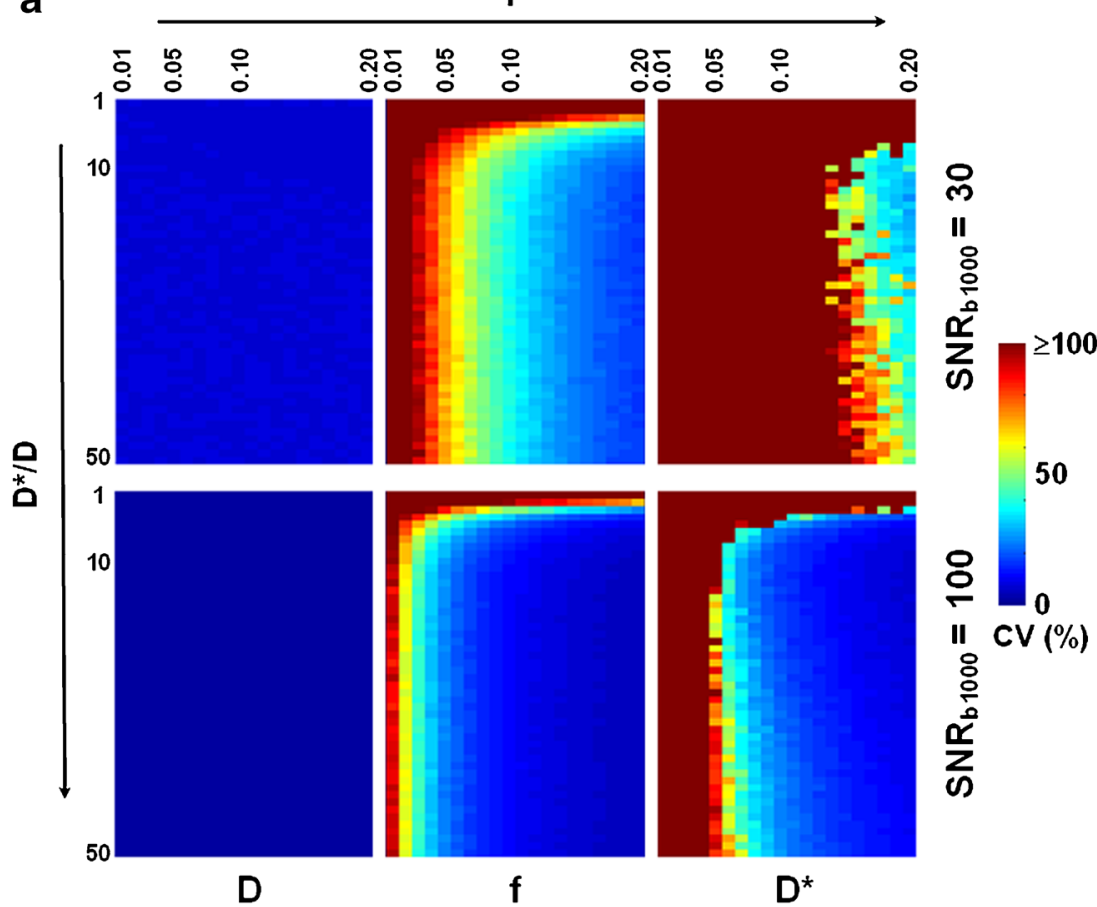

b

f

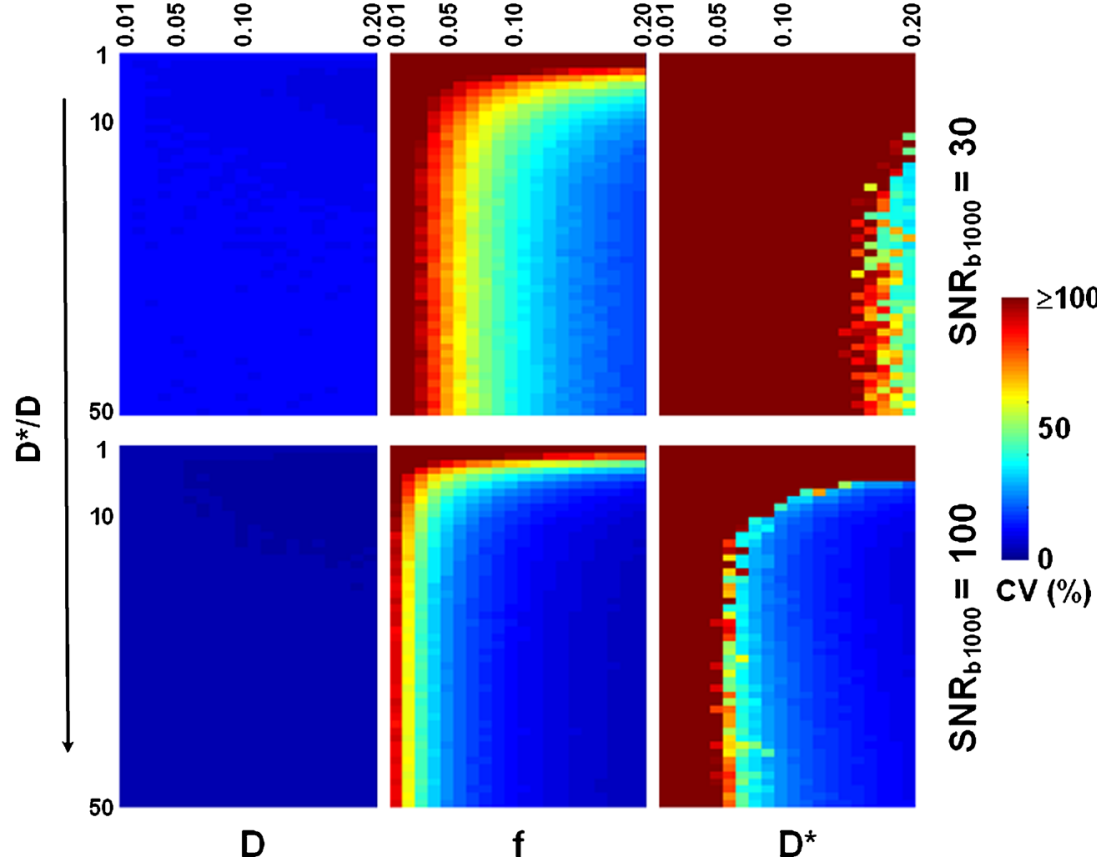


gray matter, white matter, and cerebrospinal fluid. Gray matter mask was created at a threshold of 0.9. To mitigate partial volume effect, white matter mask was created at a threshold of 0.9 after the original probability map was smoothed by a three-dimensional Gaussian kernel with a full-width-halfmaximum of $3 \mathrm{~mm}$ [22]. The abovementioned maps $\left(\mathrm{CBF}_{\mathrm{ASL}}\right.$, $\left.\mathrm{CBV}_{\mathrm{DSC}}, \mathrm{D}, \mathrm{f}, \mathrm{D}^{*}, \mathrm{SNR}_{\mathrm{b} 1000}\right)$ and masks were all coregistered to the local template for subsequent comparison and analysis.

According to [28], the product of $\mathrm{f}$ and $\mathrm{D}^{*}$ is proportional to $\mathrm{CBF}$, whereas $\mathrm{f}$ is linearly associated with $\mathrm{CBV}$ by definition. As such, we examined the correlation between $\mathrm{fD}^{*}$ and $\mathrm{CBF}_{\mathrm{ASL}}$, and between $\mathrm{f}$ and $\mathrm{CBV}_{\mathrm{DSC}}$, on a voxel-wise and per-subject basis. All post-processing and data analysis were performed by using SPM (http://www.fil.ion.ucl.ac.uk/spm/) and custom-designed programs in the environment of MATL AB (The MathWorks Inc., Natick, MA, USA).

\section{Computer simulation}

Based on equation (1), a series of IVIM signals were generated with the same b-values used in MR experiment and with varied combinations of $\mathrm{f}(0.01$ to 0.20 , in steps of 0.01$)$, $\mathrm{D}^{*}$ to $\mathrm{D}$ ratio (1 to 50 , in steps of 1 ), and $\mathrm{SNR}_{\mathrm{b} 1000}(10$ to 100 , in steps of 10). The $\mathrm{D}$ was assumed to be $0.8 \times 10^{-3} \mathrm{~mm}^{2} / \mathrm{s}$ for gray matter, $1.2 \times 10^{-3} \mathrm{~mm}^{2} / \mathrm{s}$ for axial diffusion in white matter, and $0.4 \times 10^{-3} \mathrm{~mm}^{2} / \mathrm{s}$ for radial diffusion in white matter. Rician noise was added according to the required SNR levels. For each combination, 1,000 samples were generated for IVIM model fitting, yielding 1,000 sets of $\mathrm{D} / \mathrm{f} / \mathrm{D}^{*}$ estimates. Accuracy was assessed by comparing the mean value with the theoretical value. Precision was assessed by computing the coefficient of variation.

\section{Statistical analysis}

The correlation between modalities ( $\mathrm{fD}^{*}$ versus $\mathrm{CBF}_{\mathrm{ASL}}$ and $\mathrm{f}$ versus $\mathrm{CBV}_{\mathrm{DSC}}$ ) was assessed by the Pearson's correlation coefficient at a significance level of 0.01. Bootstrapping [29] was employed to assess the robustness of IVIM measurement. In short, a specific number of repetitions were randomly selected out of the 12 repetitions (with replacement) and then averaged. This was done for every b-value to create a sample set, which was fitted with the IVIM model to render an estimate of $\mathrm{D} / \mathrm{f} / \mathrm{D}^{*}$. On a basis of 10 estimates, coefficient of variation was computed and served as a measure of precision. The $\mathrm{R}^{2}$ value of model fitting was also recorded. The procedure was carried out for repetitions of $8,6,4$, and 2 . The different numbers of repetitions can be related to SNR based on their square roots. The IVIM images obtained with $b=1$, $000 \mathrm{~s} / \mathrm{mm}^{2}$ were computed for their standard deviation and mean across the 12 repetitions. The ratio of mean to standard deviation was used as an estimate of SNR $\left(\mathrm{SNR}_{\mathrm{b} 1000)}\right)$.

\section{Results}

Computer simulations

Figure 1 shows the variance of $\mathrm{D} / \mathrm{f} / \mathrm{D}^{*}$ estimates obtained with varied combinations of $\mathrm{f}$ and $\mathrm{D}^{*}$ to $\mathrm{D}$ ratio, at two levels of $\mathrm{SNR}_{\mathrm{b} 1000}$ (30 and 100). Overall, the variance of $\mathrm{f}$ and $\mathrm{D}^{*}$ is smaller in gray matter (Fig. 1a) than in white matter (Fig. 1b), decreasing with the increase in $f, D^{*}$ to $D$ ratio, and SNR. The variance of $D$ also decreases with the increase in SNR but is largely independent of $\mathrm{f}$ and $\mathrm{D}^{*}$ to $\mathrm{D}$ ratio.

Figure 2 shows the comparison of $\mathrm{D} / \mathrm{f} / \mathrm{D}^{*}$ estimates and their theoretical values. The estimate of $\mathrm{D}$ has high accuracy (error $<5 \%$ ) regardless of SNR, gray/white matter, and axial/radial diffusion. In contrast, $f$ and $\mathrm{D}^{*}$ are overestimated at low SNR and gradually converge to the theoretical values when SNR increases. As compared with gray matter, white matter, particularly the radial diffusion, demands higher SNR to achieve a comparable level of accuracy. Again, variance decreases with the increase of SNR (see the error bars that indicate the standard deviation of 1,000 estimates). For a given SNR level, the variance is notably smaller for $\mathrm{f}$ than for $D^{*}$. Note that in white matter, the coefficient of variation of $\mathrm{D}^{*}$ is greater than $100 \%$ even when $\mathrm{SNR}_{\mathrm{b} 1000}=100$.
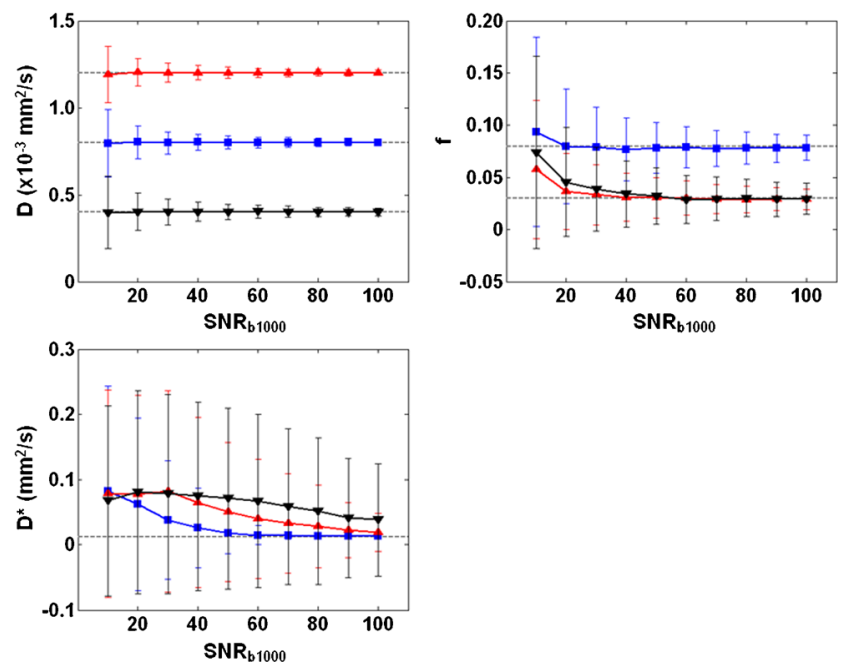

Fig. 2 Simulated accuracy of IVIM-derived diffusion coefficient (D), blood volume fraction (f), and pseudo-diffusion coefficient (D*). Gray matter (blue squares) and white matter (red upward triangles $=$ axial diffusion in white matter, black downward triangles $=$ radial diffusion in white matter) are shown separately. D was assumed to be $0.8 \times 10^{-3}$, $1.2 \times 10^{-3}$, and $0.4 \times 10^{-3} \mathrm{~mm}^{2} / \mathrm{s}$ for gray matter, axial diffusion in white matter, and radial diffusion in white matter, respectively. Gray matter and white matter were assumed to have an $\mathrm{f}$ of 0.08 and 0.03 , respectively, and the same $\mathrm{D}^{*}\left(1.2 \times 10^{-2} \mathrm{~mm}^{2} / \mathrm{s}\right)$ for simplicity. Gray dotted lines indicate the assigned values. Signal-to-noise ratio was defined at $b=$ $1,000 \mathrm{~s} / \mathrm{mm}^{2}\left(\mathrm{SNR}_{\mathrm{b} 1000}\right)$ 
Experimental SNR and parametric estimates

Figure 3 shows the typical $\mathrm{SNR}_{\mathrm{b} 1000}$ obtained with our experimental setting. $\mathrm{SNR}_{\mathrm{b} 1000}$ is higher in the cortical gray matter than in the deep gray matter partly as a consequence of coil sensitivity. For white matter, $\mathrm{SNR}_{\mathrm{b} 1000}$ is also dependent on the fibre orientation with respect to diffusion encoding. In Fig. 3b, the $\mathrm{SNR}_{\mathrm{b} 1000}$ histogram is obtained based on the masks in Fig. $3 \mathrm{a}$. The average $\mathrm{SNR}_{\mathrm{b} 1000}$ is $\sim 8$ for a single measurement, and can be related to the SNR when multiple repetitions are averaged. For example, the SNR averaged over 12 repetitions is 8 times the square root of 12 , which is $\sim 30$ (one of the values we chose to demonstrate in Fig. 1).

\section{a}
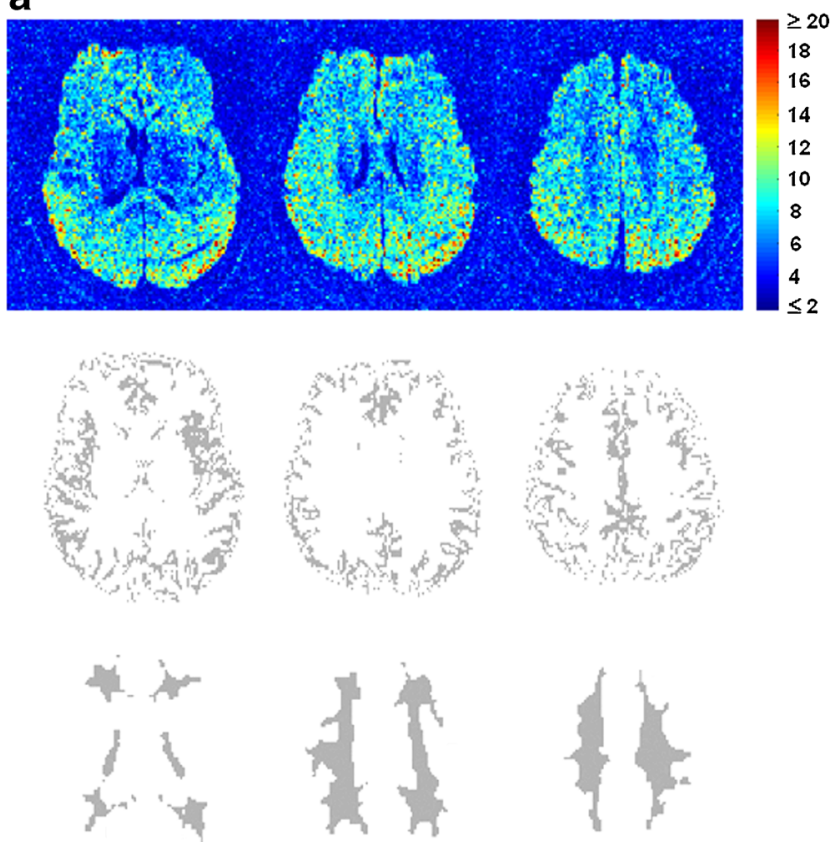

b

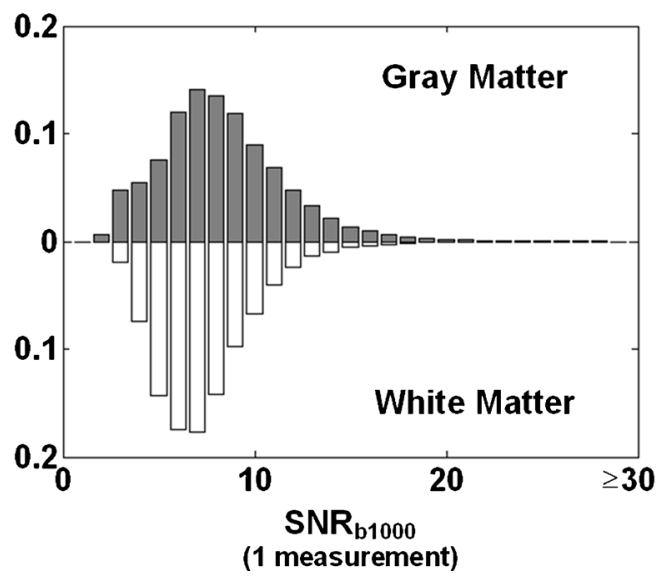

Fig. 3 Typical spatial distribution (a) and histogram (b) of signal-tonoise ratio at $b=1,000 \mathrm{~s} / \mathrm{mm}^{2}\left(\mathrm{SNR}_{\mathrm{b} 1000}\right)$ based on our imaging settings. The histogram was computed by using the masks shown in (a)

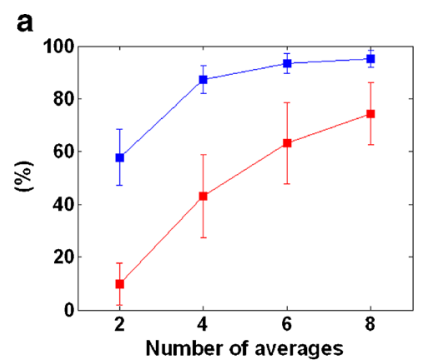

b
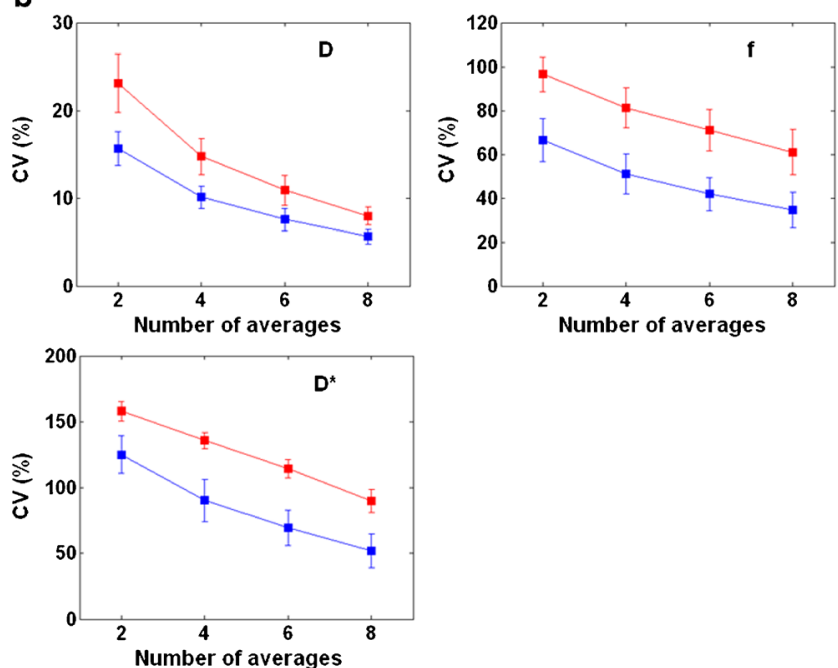

Fig. 4 (a) Percentage of voxels in which $\mathrm{R}^{2}$ of IVIM model fitting is above 0.9. (b) Variance of IVIM-derived diffusion coefficient (D), blood volume fraction (f), and pseudo-diffusion coefficient ( $\left.\mathrm{D}^{*}\right)$. On a persubject basis, 10 bootstrap samples were generated (see Materials and Methods), from which coefficient of variation (CV) was computed to assess variance and mean $\mathrm{R}^{2}$ was computed. Gray matter (blue lines) and white matter (red lines) are shown separately. The error bars indicate the standard deviation across 18 healthy volunteers

Figure 4 summarizes the parametric estimates obtained from experimental data. Error bars indicate the standard deviation across 18 subjects. Note that $\mathrm{D} / \mathrm{f} / \mathrm{D}^{*}$ were derived for three directions of diffusion encoding separately and then averaged, which reduces variance by a factor equal to the square root of 3. Figure 4 a shows the percentage of voxels where the $\mathrm{R}^{2}$ values of model fitting are higher than 0.9 . The percentage increases with the number of averages, and is consistently higher in gray matter than in white matter. Figure $4 \mathrm{~b}$ shows
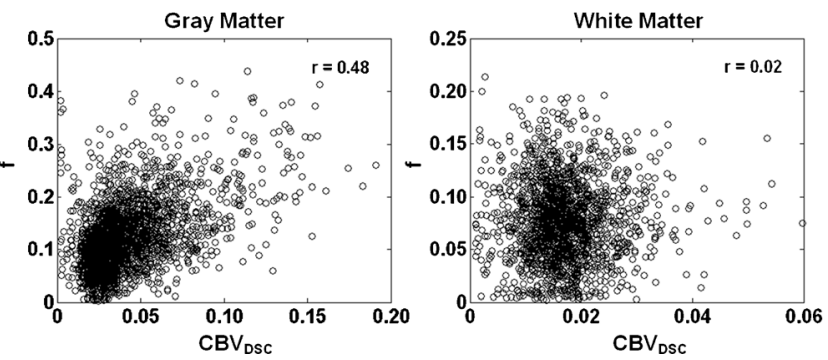

Fig. 5 Voxel-wise comparison between IVIM-derived blood volume fraction (f) and DSC-derived cerebral blood volume $\left(\mathrm{CBV}_{\mathrm{DSC}}\right)$. Data are from a representative healthy volunteer. Each circle denotes a voxel 

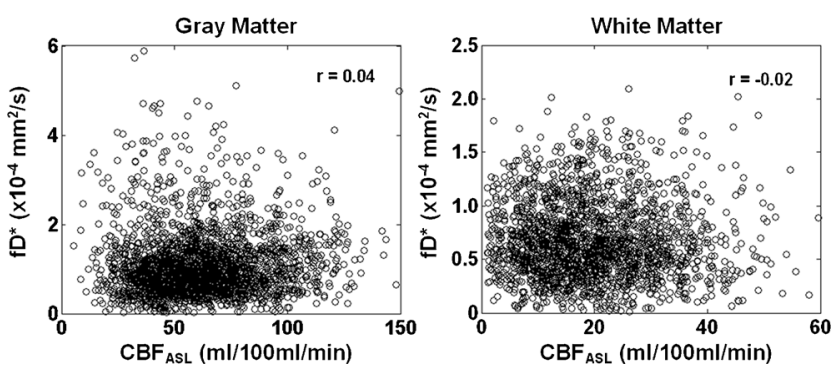

Fig. 6 Voxel-wise comparison between IVIM-derived blood flow estimate (the product of blood volume fraction $\mathrm{f}$ and pseudo-diffusion coefficient $\left.\mathrm{D}^{*}\right)$ and ASL-derived cerebral blood flow $\left(\mathrm{CBF}_{\mathrm{ASL}}\right)$. Data is from the same healthy volunteer as shown in Fig. 5. Each circle denotes a voxel

the variance in the estimates of $\mathrm{D} / \mathrm{f} / \mathrm{D}^{*}$. The estimates have lower variance (i.e., higher precision) in gray matter than in white matter. By extrapolating the plots in Fig. $4 \mathrm{~b}$ (for number of averages $=12$ ), the variance of $\mathrm{f} / \mathrm{D}^{*}$ estimates is $\sim 25 \%$ / $30 \%$ in gray matter and $\sim 50 \% / 60 \%$ in white matter, which is in a reasonable agreement with the numerical simulations shown in Figs. 1 and 2.

\section{Cross-modal comparison}

Figure 5 shows the comparison between $\mathrm{f}$ and the $\mathrm{DSC}_{\mathrm{CBV}}$ in a representative subject. Correlation is observed in gray matter $\left(\mathrm{r}=0.48, p<10^{-5}\right)$ but not in white matter $(\mathrm{r}=0.02, p=0.58)$. Note that the correlation appears to decrease when $\mathrm{DSC}_{\mathrm{CBV}}$ is below 0.02 or beyond 0.10 . Figure 6 shows the comparison between $\mathrm{fD}^{*}$ and $\mathrm{ASL}_{\mathrm{CBF}}$ in the same subject shown in Fig. 5. Correlation is absent regardless of gray/white matter, presumably due to the large variance (i.e., low precision) in the $\mathrm{D}^{*}$ estimate. These observations are highly consistent across subjects (f versus $\mathrm{DSC}_{\mathrm{CBV}}$ : $\mathrm{r}=0.29-0.48$ in gray matter, $-0.02-$ 0.05 in white matter; $\mathrm{fD}^{*}$ versus $\mathrm{ASL}_{\mathrm{CBF}}: \mathrm{r}=-0.01-0.12$ in gray matter, $-0.06-0.04$ in white matter). Table 1 summarizes the mean and standard deviation of $\mathrm{D} / \mathrm{f} / \mathrm{D}^{*}, \mathrm{DSC}_{\mathrm{CBV}}$, and $\mathrm{ASL}_{\mathrm{CBF}}$.

\section{Discussion}

Of the two IVIM-derived perfusion indexes, blood volume fraction $f$ is notably more robust than pseudo-diffusion coefficient $\mathrm{D}^{*}$ (Figs. 1, 2, and $4 \mathrm{~b}$ ). Both $\mathrm{f}$ and $\mathrm{D}^{*}$ can be better estimated when blood volume or blood flow is sufficiently large. The threshold, however, is dependent upon SNR and for white matter, also upon the fibre orientation with respect to the direction of diffusion encoding. Based on our imaging setting (particularly, the b-values and number of averages), $\mathrm{SNR}_{\mathrm{b} 1000}$ is $\sim 30$ and with which $\sim 10 \%$ error in $\mathrm{f}$ and $>100 \%$ overestimation in $\mathrm{D}^{*}$ are expected (Fig. 2). On the other hand, diffusion coefficient $\mathrm{D}$ can be estimated with high accuracy $($ error $<5 \%$ ) and precision (variance $<10 \%$ ) irrespective of gray/white matter. This suggests that perfusion has a negligible contribution to Eq. (1) when $b \geq 400 \mathrm{~s} / \mathrm{mm}^{2}$ and it is appropriate to use the two-step fitting for the IVIM model. It is worth noting that IVIM-derived indexes are highly variable when $D^{*}$ to $\mathrm{D}$ ratio is below 10 (Fig. 1). Given that $\mathrm{D}$ can be reliably measured in a wide range of $f$ and $\mathrm{D}^{*}$ and that low $\mathrm{D}^{*} / \mathrm{D}$ ratio is most likely due to low D*, IVIM imaging may not be applicable to organs where blood flow is slow (e.g., the prostate). On the other hand, several recent studies [30-33] managed to differentiate cancer pathologies in various organs by using IVIM-derived perfusion indexes. However, it may not be straightforward to generalize findings from organ to organ considering the different microvasculature and/ or flow rate in different organs.

Cross-modal comparison reveals a statistical correlation between $\mathrm{f}$ and $\mathrm{CBV}_{\text {DSC }}$ in gray matter $(\mathrm{r}=0.29-0.48$, $\left.p<10^{-5}\right)$, but not in white matter. IVIM model is not robust when $\mathrm{CBV}$ is low $\left(\mathrm{CBV}_{\mathrm{DSC}}<0.02\right)$. The correlation also drops when $\mathrm{CBV}_{\mathrm{DSC}}$ is beyond 0.10 because these voxels are likely to contain large vessels. Large vessels usually do not comply with the assumption of randomly oriented vasculature. Coherent flow leads to phase accumulation (not signal decay) and in its presence, intravascular diffusion will be attributed to D instead of D*. Taken together, f will be subject to misestimate and low precision. Wirestam et al [21] also reported a correlation between $\mathrm{CBV}_{\text {IVIM }}$ and $\mathrm{CBV}_{\mathrm{DSC}}\left(\mathrm{r}=0.563, p<10^{-5}\right)$, but two major differences should be noted between their study and ours. First, their comparison was based on regions of interest, presumably due to limited SNR (36 b-values, but only one measurement). Second, they pooled the regions of interest in gray/white matter and across subjects for correlation analysis. The inherent difference between gray matter and white matter could have inflated and dominated the computed correlation. By contrast, our comparison was on a per-subject

Table 1 Measured IVIM indexes, $\mathrm{DSC}_{\mathrm{CBV}}$, and $\mathrm{ASL}_{\mathrm{CBF}}$. Diffusion coefficient (D), blood volume fraction (f), and pseudo-diffusion coefficient (D*) were derived based on the data of 12 averages. Values are expressed as mean \pm standard deviation (across 18 subjects)

\begin{tabular}{llllll}
\hline & $\begin{array}{l}\mathrm{D} \\
\left(\times 10^{-3} \mathrm{~mm}^{2} / \mathrm{s}\right)\end{array}$ & $\mathrm{f}$ & $\begin{array}{l}\mathrm{D}^{*} \\
\left(\times 10^{-3} \mathrm{~mm}^{2} / \mathrm{s}\right)\end{array}$ & $\begin{array}{l}\mathrm{DSC} \mathrm{CBV} \\
(\mathrm{ml} / 100 \mathrm{ml})\end{array}$ & $\begin{array}{l}\mathrm{ASL} \\
(\mathrm{ml} / 100 \mathrm{ml} / \mathrm{min})\end{array}$ \\
\hline Gray matter & $0.84 \pm 0.05$ & $0.14 \pm 0.02$ & $8.2 \pm 0.9$ & $4.4 \pm 0.5$ & $62.8 \pm 4.3$ \\
White matter & $0.77 \pm 0.04$ & $0.07 \pm 0.01$ & $7.9 \pm 0.9$ & $1.9 \pm 0.2$ & $23.5 \pm 2.0$ \\
\hline
\end{tabular}


and voxel-wise basis, and was performed for gray matter and white matter separately. Our analysis should more correctly reflect the correlation between modalities, and is more applicable for per-measurement assessment. In addition, our data showed no correlation between $\mathrm{fD}^{*}$ and $\mathrm{CBF}_{\mathrm{ASL}}$. This is likely because of the large variance (i.e., low precision) in $\mathrm{D}^{*}$ estimate (Figs. 1, 2, and 4b). As mentioned previously, the $\mathrm{D}^{*}$ estimate is more SNR-demanding than the $\mathrm{f}$ estimate. Based on computer simulation, Pekar et al [24] also pointed out the SNR issue in IVIM imaging, but did not address the relationship between SNR and f/D*.

IVIM imaging does not rely on tracer delivery, which makes it a potential candidate for measuring slow or delayed perfusion such as in white matter. Unfortunately, our data showed substantial errors and variability in the IVIMderived perfusion indexes in white matter. In particular, $\mathrm{D}^{*}$ can be overestimated by $300 \%$ along with low precision (coefficient of variation $\sim 60 \%)$; $\mathrm{f}$ is relatively accurate $(\sim 10 \%$ error), but its variability is still large (coefficient of variation $\sim 50 \%$ ). A potential application to take advantage of this tracer-free feature might be $f$ measurement in tumours with blood-brain-barrier disruption in which case contrast agent leaks into interstitial space, leading to complex interplay between $\mathrm{T} 1$ and $\mathrm{T} 2 *$ effects. $\mathrm{CBV}_{\mathrm{DSC}}$ measured under such a circumstance has been known to be subject to error unless the $\mathrm{T} 1$ effect can be removed or corrected for [34].

There are a few limitations in this study. First, the data presented are based on healthy volunteers in their 20s and $30 \mathrm{~s}$. Nevertheless, our results provide quantitative assessment that helps parameter adjustment for IVIM imaging. Second, we did not convert $f$ and $D^{*}$ to $C B V$ and $C B F$ quantities. The purpose of this study is to evaluate the robustness of IVIMderived perfusion indexes. According to [28], the calculation of $\mathrm{CBV}$ and $\mathrm{CBF}$ primarily relies on $\mathrm{f}$ and $\mathrm{D}^{*}$, whose precision and accuracy can be inferred from our data. Third, in our fitting we did not consider non-Gaussian diffusion [35] that can be characterized by including a diffusion kurtosis coefficient to Eq. (1). Given that the kurtosis term contributes at high b-values (usually beyond $1,000 \mathrm{~s} / \mathrm{mm}^{2}$ ), our IVIM imaging was based on relatively low b-values and should not include notable kurtosis effect. The suitability of our model fitting without the kurtosis term can also be revealed by the high percentage of voxels where $\mathrm{R}^{2}$ is above 0.9 with the IVIM model ( $\sim 95 \%$ in gray matter and $\sim 85 \%$ in white matter). The expense of using the extended model is adding an additional degree of freedom to the nonlinear least-squares fitting that is already SNR demanding. It is noteworthy that in addition to kurtosis, non-Gaussian diffusion has also been investigated with two-exponential model (slow vs. fast diffusing compartments or intra- vs. extra-cellular compartments) $[36,37]$ and stretched exponential model (multiple compartments and thus continuous distribution of diffusion coefficients) [38].
In summary, we have numerically and experimentally assessed the robustness of IVIM imaging in measuring cerebral perfusion indexes. A minimum $\mathrm{SNR}_{\mathrm{b} 1000}$ of 30 is recommended such that reliable $\mathrm{f}$ can be obtained as a noninvasive measure of cerebral blood volume. However, $\mathrm{D}^{*}$ has limited robustness and should be interpreted with caution.

Acknowledgments The scientific guarantor of this publication is WenChau Wu. This study has received funding by Taiwan National Science Council. No complex statistical methods were necessary for this paper. Institutional Review Board approval was obtained.

Written informed consent was obtained from all subjects (patients) in this study. Methodology: prospective, performed at one institution

Open Access This article is distributed under the terms of the Creative Commons Attribution Noncommercial License which permits any noncommercial use, distribution, and reproduction in any medium, provided the original author(s) and the source are credited.

\section{References}

1. Wiest R, von Bredow F, Schindler K et al (2006) Detection of regional blood perfusion changes in epileptic seizures with dynamic brain perfusion CT-a pilot study. Epilepsy Res 72:102-110

2. Tosun D, Mojabi P, Weiner MW, Schuff N (2010) Joint analysis of structural and perfusion MRI for cognitive assessment and classification of Alzheimer's disease and normal aging. Neuroimage 52:186197

3. Heiss WD, Raab P, Lanfermann H (2011) Multimodality assessment of brain tumors and tumor recurrence. J Nucl Med 52:1585-1600

4. Rosen BR, Belliveau JW, Vevea JM, Brady TJ (1990) Perfusion imaging with NMR contrast agents. Magn Reson Med 14:249-265

5. Detre JA, Leigh JS, Williams DS, Koretsky AP (1992) Perfusion imaging. Magn Reson Med 23:37-45

6. Le Bihan D, Breton E, Lallemand D, Aubin ML, Vignaud J, LavalJeantet M (1988) Separation of diffusion and perfusion in intravoxel incoherent motion MR imaging. Radiology 168:497-505

7. Meier P, Zierler KL (1954) On the theory of the indicator-dilution method for measurement of blood flow and volume. J Appl Physiol 6:731-744

8. Ostergaard L, Weisskoff RM, Chesler DA, Gyldensted C, Rosen BR (1996) High resolution measurement of cerebral blood flow using intravascular tracer bolus passages. Part I: Mathematical approach and statistical analysis. Magn Reson Med 36:715-725

9. Olivot JM, Mlynash M, Thijs VN et al (2009) Optimal Tmax threshold for predicting penumbral tissue in acute stroke. Stroke 40:469475

10. Marckmann P, Skov L, Rossen K et al (2006) Nephrogenic systemic fibrosis: suspected causative role of gadodiamide used for contrastenhanced magnetic resonance imaging. J Am Soc Nephrol 17:23592362

11. Chrysochou C, Buckley DL, Dark P, Cowie A, Kalra PA (2009) Gadolinium-enhanced magnetic resonance imaging for renovascular disease and nephrogenic systemic fibrosis: critical review of the literature and UK experience. J Magn Reson Imaging 29:887-894

12. Wu WC, St Lawrence KS, Licht DJ, Wang DJ (2010) Quantification issues in arterial spin labeling perfusion magnetic resonance imaging. Top Magn Reson Imaging 21:65-73

13. Petersen ET, Lim T, Golay X (2006) Model-free arterial spin labeling quantification approach for perfusion MRI. Magn Reson Med 55: 219-232 
14. Wang J, Alsop DC, Song HK et al (2003) Arterial transit time imaging with flow encoding arterial spin tagging (FEAST). Magn Reson Med 50:599-607

15. Guiu B, Petit JM, Capitan Vet al (2012) Intravoxel incoherent motion diffusion-weighted imaging in nonalcoholic fatty liver disease: a 3.0T MR study. Radiology 265:96-103

16. Klauss M, Gaida MM, Lemke A et al (2013) Fibrosis and pancreatic lesions: counterintuitive behavior of the diffusion imaging-derived structural diffusion coefficient d. Invest Radiol 48:129-133

17. Chandarana H, Kang SK, Wong S et al (2012) Diffusion-weighted intravoxel incoherent motion imaging of renal tumors with histopathologic correlation. Invest Radiol 47:688-696

18. Sigmund EE, Cho GY, Kim S et al (2011) Intravoxel incoherent motion imaging of tumor microenvironment in locally advanced breast cancer. Magn Reson Med 65:1437-1447

19. Neil JJ, Bosch CS, Ackerman JJ (1994) An evaluation of the sensitivity of the intravoxel incoherent motion (IVIM) method of blood flow measurement to changes in cerebral blood flow. Magn Reson Med 32:60-65

20. Federau C, Maeder P, O'Brien K, Browaeys P, Meuli R, Hagmann P (2012) Quantitative measurement of brain perfusion with intravoxel incoherent motion MR imaging. Radiology 265:874-881

21. Wirestam R, Borg M, Brockstedt S, Lindgren A, Holtas S, Stahlberg F (2001) Perfusion-related parameters in intravoxel incoherent motion MR imaging compared with CBV and CBF measured by dynamic susceptibility-contrast MR technique. Acta Radiol 42:123-128

22. Wu WC, Lin SC, Wang DJ, Chen KL, Li YD (2013) Measurement of cerebral white matter perfusion using pseudocontinuous arterial spin labeling 3 T magnetic resonance imaging-an experimental and theoretical investigation of feasibility. PLoS ONE 8:e82679

23. Zhang Q, Wang YX, Ma HT, Yuan J (2013) Cramer-Rao bound for Intravoxel Incoherent Motion Diffusion Weighted Imaging fitting. Conf Proc IEEE Eng Med Biol Soc 2013:511-514

24. Pekar J, Moonen CT, van Zijl PC (1992) On the precision of diffusion/perfusion imaging by gradient sensitization. Magn Reson Med 23:122-129

25. Garcia DM, de Bazelaire C, Alsop D (2005) Pseudo-continuous flow driven adiabatic inversion for arterial spin labeling. Proc Int Soc Magn Reson Med 13:37

26. Wu WC, Jiang SF, Yang SC, Lien SH (2011) Pseudocontinuous arterial spin labeling perfusion magnetic resonance imaging-a normative study of reproducibility in the human brain. Neuroimage $56: 1244-1250$

27. Calamante F, Thomas DL, Pell GS, Wiersma J, Turner R (1999) Measuring cerebral blood flow using magnetic resonance imaging techniques. J Cereb Blood Flow Metab 19:701-735

28. Le Bihan D, Turner R (1992) The capillary network: a link between IVIM and classical perfusion. Magn Reson Med 27:171-178

29. Efron B, Tibshirani R (1993) An Introduction to the Bootstrap. Chapman \& Hall, New York

30. Iima M, Reynaud O, Tsurugizawa T et al (2014) Characterization of glioma microcirculation and tissue features using intravoxel incoherent motion magnetic resonance imaging in a rat brain model. Invest Radiol 49:485-490

31. Woo S, Lee JM, Yoon JH, Joo I, Han JK, Choi BI (2014) Intravoxel incoherent motion diffusion-weighted MR imaging of hepatocellular carcinoma: correlation with enhancement degree and histologic grade. Radiology 270:758-767

32. Lai V, Li X, Lee VH, Lam KO, Chan Q, Khong PL (2013) Intravoxel incoherent motion MR imaging: comparison of diffusion and perfusion characteristics between nasopharyngeal carcinoma and postchemoradiation fibrosis. Eur Radiol 23:2793-2801

33. Kang KM, Lee JM, Yoon JH, Kiefer B, Han JK, Choi BI (2014) Intravoxel incoherent motion diffusion-weighted MR imaging for characterization of focal pancreatic lesions. Radiology 270:444-453

34. Boxerman JL, Schmainda KM, Weisskoff RM (2006) Relative cerebral blood volume maps corrected for contrast agent extravasation significantly correlate with glioma tumor grade, whereas uncorrected maps do not. AJNR Am J Neuroradiol 27:859-867

35. Jensen JH, Helpern JA (2010) MRI quantification of non-Gaussian water diffusion by kurtosis analysis. NMR Biomed 23:698-710

36. Mulkern RV, Gudbjartsson H, Westin CF et al (1999) Multicomponent apparent diffusion coefficients in human brain. NMR Biomed 12:51-62

37. Clark CA, Le Bihan D (2000) Water diffusion compartmentation and anisotropy at high b values in the human brain. Magn Reson Med 44: 852-859

38. Bennett KM, Schmainda KM, Bennett RT, Rowe DB, Lu H, Hyde JS (2003) Characterization of continuously distributed cortical water diffusion rates with a stretched-exponential model. Magn Reson Med 50:727-734 